ИЗВЕСТИЯ АКАДЕМИИ НАУК ЭСТОНСКОИ ССР. ФИЗИКА МАТЕМАТИКА

PROCEEDINGS OF THE ACADEMY OF SCIENCES OF THE ESTONIAN SSR. PHYSICS * MATHEMATICS

$1987,36,3$

удК $519.642 .2+517.983 .246$

Ю. КНЯЗИХИН

\title{
К СХОДИМОСТИ МЕТОДА ЗЕЙЕЛЯ РЕШЕНИЯ ОПЕРАТОРНЫХ УРАВНЕНИЙ
}

\section{(Представил Г. Вайникко)}

1. Пусть $H$ - вещественное гильбертово пространство, а $B-$ самосопряженный ограниченный положительно определенный оператор:

$$
B: H \rightarrow H, \quad(B x, x) \geqslant m(x, x), \quad m>0, \quad x \in H .
$$

Рассмотрим уравнение

$$
B x=b .
$$

Для решения уравнения (2) применим метод Зейделя

$$
x_{n+1}=-\left(B_{1}+B_{2}\right)^{-1} B_{2}^{*} x_{n}+\left(B_{1}+B_{2}\right)^{-1} b, \quad x_{0} \in H .
$$

Здесь $B=B_{1}+B_{2}+B_{2}{ }^{*}$, а $B_{1}$ - ограниченный положительно определенный оператор,

$$
\left(B_{1} x, x\right) \geqslant m_{1}(x, x), \quad m_{1}>0, \quad x \in H .
$$

Очевидно, что если спектральный радиус $\mathrm{Q}(C)$ оператора $C=$ $=\left(B_{1}+B_{2}\right)^{-1} B_{2}{ }^{*}$ меньше 1 , то метод (3) сходится к решению уравнения (2) при любом начальном приближении $x_{0} \in H$. В монографии [ $\left.{ }^{1}\right]$ (c. 103) дана следующая оценка спектрального радиуса @ $(C)$ :

$$
\varrho(C) \leqslant 1-\left[\beta_{0}-\left(\beta_{0}^{2}-m m_{1}\right)^{1 / 2}\right]\left\|B_{1}+B_{2}\right\|^{-1}<1,
$$

где $\beta_{0}=\sup _{\|x\|=1}\left|\left(\left(B_{1}+B_{2}\right) x, x\right)\right|$.

В предлагаемой работе мы уточним эту оценку в случае, когда $B_{1}$ и $B_{2}$ самосопряженные операторы. Рассмотрим непрерывную функцию

$$
m(t)=\inf _{\|x\|=1}\left(\left(B_{1}+B_{2}+t B_{2}\right) x, x\right)
$$

и множество $M=\{t>0: m(t)>0$ и $m(-t)>0\}$. Определим операторы $K_{1}, K_{2}$ и $K$ по правилу

$$
K_{1}=B_{1}+B_{2}-t B_{2}, \quad K_{2}=t B_{2}, \quad K=B_{1}+B_{2}+t B_{2}^{*}, \quad t \in M .
$$

Нетрудно убедиться, что спектральный радиус $\varrho(t)$ оператора $\left(K_{1}+K_{2}\right)^{-1} K_{2}^{*}$ связан с $\mathrm{Q}(C)$ при помощи равенства

$$
|t| \varrho(C)=\varrho(t) .
$$

Для оценки $\varrho(t)$ используем неравенство (5). После несложных преобразований получим

$\varrho(C)=|t|^{-1} \varrho(t) \leqslant|t|^{-1}\left\{1-\left[\beta_{0}-\left(\beta_{0}^{2}-m(t) m(-t)\right)^{1 / 2}\right]\left\|B_{1}+B_{2}\right\|^{-1}\right\}$.

Последнее неравенство выполняется для всех $|t| \in M$. Исходя из соображений непрерывности, заключаем, что неравенство (6) выполнено и 
для любого $t \in \bar{M}$ (здесь $\bar{M}-$ замыкание множества $M$ ). Отсюда получаем

$$
\mathrm{Q}(C) \leqslant \min _{t \in \bar{M}} v(t),
$$

где $v(t)=|t|^{-1}\left\{1-\left[\beta_{0}-\left(\beta_{0}^{2}-m(t) m(-t)\right)^{1 / 2}\right]\left\|B_{1}+B_{2}\right\|^{-1}\right\}$.

Т е о р е а 1. Нмеет место соотношение

$$
\mathrm{Q}(C)=1 / t^{*}=\min _{t \in \bar{M}} v(t)<1,
$$

где $t^{*}$ есть единственный в $\bar{M}$ корень уравнения

$$
m(t) m(-t)=0, \quad t \in \bar{M} .
$$

Доказательство. Рассмотрим семейство операторов $A(t)=$ $=B_{1}+B_{2}+t B_{2}{ }^{*}$. В силу (1) и (2) оператор $\left(B_{1}+B_{2}\right)$ обратим. Поэтому $A(t)=\left(B_{1}+B_{2}\right)\left(E+t\left(B_{1}+B_{2}\right)^{-1} B_{2}^{*}\right)=\left(B_{1}+B_{2}\right)(E+t C)$. Для всех $|t|<$ $<1 / \mathrm{Q}(C)$ оператор $E+t C$ обратим, а следовательно, для этих значений обратим и оператор $A(t)$.

Далее, из условия (1) следует, что оператор $B=B_{1}+B_{2}+B_{2}{ }^{*}$ обратим. Поэтому $A(t)=B_{1}+B_{2}+B_{2}{ }^{*}+(t-1) B_{2}{ }^{*}=B\left(E+(t-1) B^{-1} B_{2}{ }^{*}\right)$. Отметим, что операторы $E+t C$ и $E+(t-1) B^{-1} B_{2}{ }^{*}$ при каждом фиксированном $t$ либо обратимы, либо необратимы одновременно. Отсюда следует, что оператор $E+(t-1) B^{-1} B_{2}{ }^{*}$ будет обратим, как только

$$
-1-1 / \mathrm{\varrho}(C)<t-1<-1+1 / \mathrm{Q}(C) \text {. }
$$

Из неравенства (5) следует, что $-1+1 / \varrho(C)>0$, а из ограниченности оператора $B^{-1} B_{2}{ }^{*}$ вытекает неравенство $|t-1| \varrho\left(B^{-1} B_{2}{ }^{*}\right)<1$. Отсюда следует, что при $|t|<1 / \varrho(C)$ оператор $E+(t-1) B^{-1} B_{2}^{*}$ положительно определен:

$$
\left(\left(E+(t-1) B^{-1} B_{2}^{*} x, x\right) \geqslant r(t)(x, x), r(t)>0,|t|<1 / \varrho(C) .\right.
$$

Нетрудно увидеть, что оператор $A(t)$ при $|t|<1 / \mathrm{Q}(C)$ будет положительно определенным в гильбертовом пространстве $H_{B}$ со скалярным произведением $(x, y)_{B}=\left(B^{-1} x, x\right)$. Следовательно, оператор $A(t)$ будет положительно определенным и в пространстве $H$. Отсюда при $|t| \in M$ функция $m(t)$ строго положительна: $m(t)>0$.

Покажем теперь, что если $|t|>1 / \mathrm{Q}(C)$, то $|t| \notin M$. Предположим обратное: пусть $|t| \in M$. Из (6) следует

$$
\varrho(C) \leqslant v(t) \leqslant|t|^{-1}<\varrho(C) .
$$

Полученное противоречие доказывает, что $|t| \notin M$.

Так как $m(t)$ непрерывна, то $m(1 / \varrho(C)) m(-1 / \varrho(C))=0$. Отсюда и из (5) следует равенство $\bar{M}=[0,1 / \varrho(C)]$ и формула (7).

Теорема доказана.

2. Рассмотрим применение теоремы 1 на следующем примере. Пусть $S$ - единичная сфера в $R^{3}$, а $\Omega, \Omega^{\prime}$ - точки на этой сфере. Рассмотрим пространство $L_{2}(S)$ определенных на $S$ функций $u(\Omega)$, в котором скалярное произведение и норма определяются как

$$
(u, v)=\int_{S} u(\Omega) v(\Omega) d \Omega, \quad|u|=(u, u)^{1 / 2},
$$

где интеграл есть поверхностный интеграл 1 рода.

Определим на $L_{2}(S)$ оператор $G$, действующий по правилу

$$
(G u)(\Omega)=\lambda(4 \pi)^{-1} \int_{S} g\left(\Omega \Omega^{\prime}\right) u\left(\Omega^{\prime}\right) d \Omega^{\prime}, \quad 0<\lambda<1 .
$$


Здесь $\Omega \Omega^{\prime}$ скалярное произведение единичных векторов $\Omega$ и $\Omega^{\prime}$, а $g(\mu)$ функция, удовлетворяющая условию

$$
g(\mu) \geqslant 0, \quad \int_{-1}^{1} g(\mu) d \mu=2 .
$$

Можно показать $\left[{ }^{2}\right]$, что $\|G\|=\lambda$. Пусть

$$
\begin{aligned}
& S^{+}=\left\{(x, y, z): x^{2}+y^{2}+z^{2}=1, z \geqslant 0\right\}, \\
& S^{-}=\left\{(x, y, z): x^{2}+y^{2}+z^{2}=1, z<0\right\} .
\end{aligned}
$$

Наряду с оператором $G$ рассмотрим операторы

$$
\begin{aligned}
& \left(G^{+} u\right)(\Omega)=\lambda(4 \pi)^{-1} \int_{\mathcal{S}^{+}} g\left(\Omega \Omega^{\prime}\right) u\left(\Omega^{\prime}\right) d \Omega^{\prime}, \\
& \left(G^{-} u\right)(\Omega)=\lambda(4 \pi)^{-1} \int_{\mathcal{S}^{-}} g\left(\Omega \Omega^{\prime}\right) u\left(\Omega^{\prime}\right) d \Omega^{\prime} .
\end{aligned}
$$

Из $S=S_{1} \cup S_{2}, S_{1} \cap S_{2}=\varnothing$ следует, что $G=G^{+}+G^{-}$. Операторы $G, G^{+}$, $G^{-}$являются самосопряженными и ограниченными.

Исследование сходимости метода Зейделя применительно к решению уравнения переноса сводится $\left[{ }^{3}\right]$ к вычислению спектрального радиуса @ оператора $\left(E-G^{+}\right)^{-1} G^{-}$. Положим

$$
B_{1}=E-G^{+}-G^{-}, \quad B_{2}=G^{-}, \quad B=B_{1}+B_{2}+B_{2}^{*}=E-G^{+}+G^{-} .
$$

Очевидно, что $\left(B_{1}+B_{2}\right)^{-1} B_{2}{ }^{*}=\left(E-G^{+}\right)^{-1} G^{-}$. Поэтому для вычисления можно применить теорему 1 . Имеем

$$
\begin{gathered}
m(t)=\inf _{\|x\|=1}\left(\left(B_{1}+B_{2}+t B_{2}\right) x, x\right)= \\
=\inf _{\|x\|=1}\left((x, x)-\left(\left(G^{+}-t G^{-}\right) x, x\right)=1-\gamma(-t),\right.
\end{gathered}
$$

где $\gamma(t)=\sup _{\|x\|=1}\left(\left(G^{+}+t G^{-}\right) x, x\right)$.

Рассмотрим уравнение

$$
(1-\gamma(t))(1-\gamma(-t))=0, t \in \bar{M}=\{t \geqslant 0: \gamma(t) \leqslant 1 \text { и } \gamma(-t) \leqslant 1\} .
$$

Согласно теореме 1 , спектральный радиус @ оператора $\left(E-G^{+}\right)^{-1} G^{-}$ есть $1 / t^{*}$, где $t^{*}$ - единственный корень уравнения (9).

Т е о ре м а 2. Спектральный радиус оператора $\left(E-G^{+}\right)^{-1} G^{-}$есть $1 / t^{*}$, где $t^{*}$ - единственный положительный корень уравнения $\left\|G^{+}+t G^{-}\right\|=1$, причем функция $\left\|G^{+}+t G^{-}\right\|$монотонно возрастает при $t \geqslant 0$.

Дока з а тел ь с тво. Пусть $K \subset L_{2}(S)-$ конус неотрицательных функций. Он является нормальным и воспроизводящим $\left[{ }^{1}\right]$ (с. 76$)$. Операторы $G^{+}, G^{-}$и $G^{+}+t G^{-}(t \geqslant 0)$ являются положительными относительно конуса $K$. Из самосопряженности $G^{+}$и $G^{-}$следует неравенство

$$
\gamma(t) \leqslant \sup _{\|x\|=1}\left|\left(\left(G^{+}+t G^{-}\right) x, x\right)\right|=\left\|G^{+}+t G^{-}\right\|, \quad \forall t .
$$

Из положительности относительно конуса $K$ оператора $G^{+}+t G^{-}$при $t \geqslant 0$ следует, что спектральный радиус этого оператора является точкой его спектра. Значит при $t \geqslant 0$ имеет место равенство

$$
\gamma(t)=\left\|G^{+}+t G^{-}\right\| \text {. }
$$

Из неравенства

$$
-G^{+} x-t_{2} G^{-} x \leqslant G^{+} x+t_{1} G^{-} x \leqslant G^{+} x+t_{2} G^{-} x, \quad x \in K, \quad t_{1} \leqslant t_{2}
$$

следует $\left.{ }^{1}\right]$ (с. 79) монотонность функции $\left\|G^{+}+t G^{-}\right\|$

$$
\left\|G^{+}+t_{1} G^{-}\right\| \leqslant\left\|G^{+}+t_{2} G^{-}\right\|, \quad 0 \leqslant t_{1} \leqslant t_{2},
$$




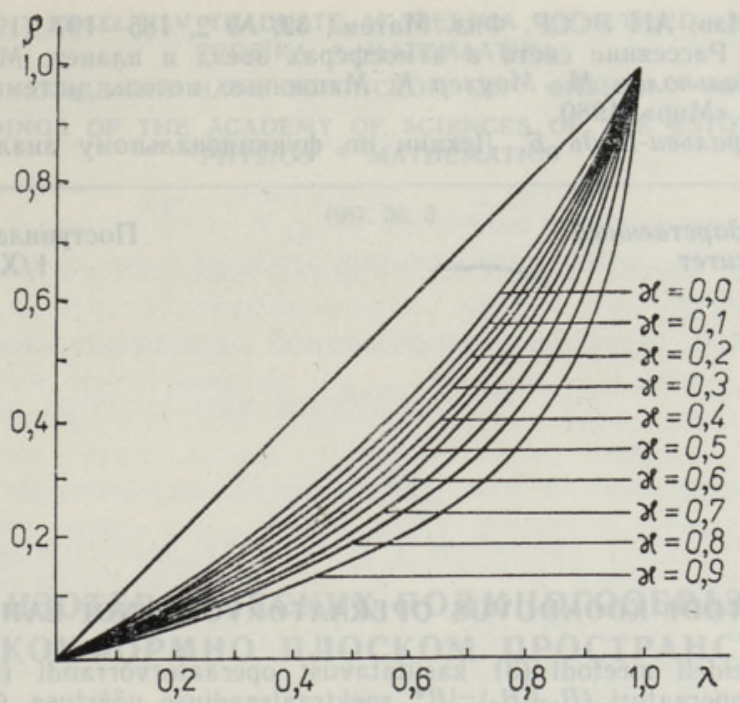

Зависимость спектрального радиуса $\varrho$ оператора $\left(E-G^{+}\right)^{-1} G^{-}$от $\lambda$ при различных значениях $x$.

а из неравенства

$$
-G^{+} x-t G^{-} x \leqslant G^{+} x-t G^{-} x \leqslant G^{+} x+t G^{-} x, \quad x \in K, \quad t \geqslant 0
$$

следует, что $\gamma(-t) \leqslant\left\|G^{+}-t G^{-}\right\| \leqslant\left\|G^{+}+t G^{-}\right\|$. Поэтому, если $\gamma(t)=$ $=\left\|G^{+}+t G^{-}\right\|=1$, то

$$
\gamma(-t) \leqslant\left\|G^{+}-t G^{-}\right\| \leqslant\left\|G^{+}+t G^{-}\right\|=1 .
$$

Следовательно, для вычисления спектрального радиуса оператора $\left(E+G^{+}\right)^{-1} G^{-}$достаточно найти корень уравнения $\left\|G^{+}+t G^{-}\right\|=1, t \geqslant 0$. Из монотонности $\gamma(t)$ при $t \geqslant 0$ и равенства $\gamma(1)=\left\|G^{+}+G^{-}\right\|=\lambda$ следует неравенство $t^{*}>1$.

Теорема доказана.

Рассмотрим параметрическое семейство функций $g(x, \mu)$, определяющих оператор $G$ (см. формулу (8))

$$
g(x, \mu)=\left(1-x^{2}\right)\left(1+x^{2}-2 x \mu\right)^{-3 / 2}, \quad \int_{-1}^{1} g(x, \mu) d \mu=1,|x|<2 .
$$

Функция $g(x, \mu)$ называется в теории переноса излучения индикатрисой рассеяния Хеньи-Гринстейна и описывает довольно широкий класс реальных законов рассеяния $\left[{ }^{4}\right]$. Параметр $x$ характеризует анизотропность рассеяния: чем ближе значение $x$ к единице, тем больше анизотропность рассеяния. Из рисунка видно, что чем больше анизотропность рассеяния «вперед» (т. е. $x>0$ ), тем быстрее сходится метод Зейделя к решению уравнения переноса.

Для решения уравнения $\left\|G^{+}+t G^{-}\right\|=1$ использовалась программа ZEROIN $\left[{ }^{5}\right]$, а для вычисления функции $\left\|G^{+}+t G^{-}\right\|$метод Келлога [ $\left.{ }^{6}\right]$.

\section{Л ИТЕ Р АТ У Р А}

1. Красносельский М. А., Вайникко Г. М., Забрейко П. П., Рутицкий Я. Б., Стеценко В. Я. Приближенное решение операторных уравнений. М., «Наука», 1969.

2. Владимиров В. С. Тр. мат. ин-та АН СССР, № 61, 1-160 (1961). 
3. Князихин Ю. Изв. АН ЭССР. Физ. Матем., 32, № 2, 185-197 (1982).

4. Соболев В. В. Рассеяние света в атмосферах звезд и планет. М., «Наука», 1972.

5. Форсайт Д., Малькольм М., Моулер К. Машинные методы математических вычислений. М., «Мир», 1980.

6. Рисс Ф., Секефальви-Надь Б. Лекции по функциональному анализу. М., «Мир», 1979.

Тартуский государственный университет

Поступила в редакцию 1/XII 1986

\section{J. KNJAZIHHIN}

\section{SEIDELI MEETODI KOONDUVUS OPERAATORVORRANDI LAHENDAMISEL}

On uuritud Seideli meetodi (3) kasutatavust operaatorvõrrandi (2) lahendamisel. Teoreem 1 annab operaatori $\left(B_{1}+B_{2}\right)^{-1} B_{2}^{*}$ spektraalraadiuse väärtuse. On vaadeldud ka arvuliste näidetega teoreeemi 1 rakendusvõimalusi atmosfäärioptikas.

\section{Yu. KNYAZIKHIN}

\section{CONVERGENCE OF THE SEIDEL ITERATIVE METHODS TO SOLVE OPERATOR EQUATION}

Let $B$ be self-conjugate positive definite (see formula (1)) operator in the real Hilbert space $H$. The operator equation (2) and Seidel iterative methods (3) are considered. Our purpose is to investigate spectral radius of operator $\left(B_{1}+B_{2}\right)^{-1} B_{2}^{*}$.

Theorem 1. The spectral radius of the operator $\left(B_{1}+B_{2}\right)^{-1} B_{2}^{*}$ is equal to $1 / t^{*}$, where $t^{*}$ is the unique solution of the equation $m(t) m(-t)=0, t \in M ;$ continuous function $m(t)$ and set $\bar{M}$ are defined by

$$
m(t)=\inf _{\|x\|=1}\left(\left(B_{1}+B_{2}+t B_{2}\right) x, x\right), \quad \bar{M}=\{t \geqslant 0 \mid m(t) \geqslant 0 \text { and } m(-t) \geqslant 0\} .
$$

The operator $\left(E-G^{+}\right)^{-1} G^{-}$which occurs in transport theory is considered in the second part of our paper. The spectral radius of that operator is expressed as reciprocal value of the unique solution of the equation $\left\|G^{+}+t G^{-}\right\|=1$.

Let the operator $G$ be defined by function (10) (the Henyey-Greenstein phase function). The Figure demonstrates the dependence of spectral radius of the $\left(E-G^{+}\right)^{-1} G^{-}$ on value $\lambda$ for some parameters $x$. 